\title{
Superficial Anastomotic Veins: Neurosurgical View Depending on 251 Craniotomies
}

\author{
Taner Tanriverdi, Hosam Al-Jehani, Nicole Poulin, Andre Olivier
}

\begin{abstract}
Background: Superficial anastomotic veins (SAVs) have been studied extensively but little attention has been paid to clinical studies. The aim of this study is to provide variations in the drainage patterns of SAVs depending on the intraoperative findings. Methods: A total of 251 craniotomies due to intractable temporal lobe epilepsy were performed between 1972 and 1987 at the Montreal Neurological Institute. The courses of the three largest SAVs including the vein of Trolard (VT), vein of Labbe (VL) and superficial Sylvian vein (SSV) were studied. Results: All three veins showed variable courses. The most common predominant vein was the combination of the VL + SSV. The VT and VL were frequently coursed at the level of the central vein and middle temporal vein, respectively. On the right hemisphere the SSV was the predominant type while the VL tended to be predominant on the left hemisphere. A combination of VL and SSV was predominant in patients with right and/or left hemispheric dominance. Conclusions: The SAVs showed considerable variation in their courses and it is difficult to define an exact pattern although some courses showed constant directions. Attention should be paid not to damage these veins since in a considerable number of cases a single dominant vein may be responsible for draining a majority of the lateral surface of cerebral hemisphere.
\end{abstract}

RÉSUMÉ: Objectif: Des veines anastomotic superficielles (SAVs) ont été étudiées intensivement mais peu d'attention a été payées aux études cliniques. Le but de cette étude est de fournir des variations des modèles de drainage des SAVs selon les résultats peropératoires. Méthode: Un total de 251 craniotomies dus à l'épilepsie temporelle insurmontable de lobe ont été exécutés entre 1972 et 1987 à l'institut neurologique de Montréal. Les cours des trois plus grands SAVs comprenant la veine de Trolard (VT), la veine de Labbe (VL) et la veine sylvian superficielle (SSV) ont été étudiés. Résultat: Chacune des trois veines montrées des cours variables. La veine prédominante la plus commune était la combinaison du VL + SSV. Les VT et les VL ont été fréquemment courus au niveau de la veine centrale et de la veine temporelle moyenne, respectivement. Sur le bon hémisphère le SSV était le type prédominant tandis que le VL tendait à être prédominant sur l'hémisphère gauche. La combinaison du VL et du SSV était prédominante dans les patients présentant la bonne et/ou gauche dominance hémisphérique. Conclusion: Les SAVs ont montré que les variations considérables de leurs cours et lui est difficile de définir un modèle exact bien que quelques cours aient montré des directions constantes. L'attention devrait être prêtée pour ne pas endommager ces veines puisque dans un nombre considérable de cas une veine dominante simple peut être responsable de vidanger une majorité de la surface latérale de l'hémisphère cérébral.

Can. J. Neurol. Sci. 2009; 36: 65-71

Although several studies, including cadaveric ${ }^{1-8}$ and imaging, ${ }^{9-15}$ exist in the literature, little attention has been paid to clinical studies ${ }^{16}$ of the superficial anastomotic veins (SAVs), damage to which may cause catastrophic complications even after successful surgery. It is generally agreed that these veins constitute an irregular network and show variations in size and course that make it difficult to define a normal pattern. Classically, there are the largest SAVs which lie along the lateral surface of the brain. The vein of Trolard (VT), or superior anastomotic vein, is the largest vein crossing the cortical surface of the frontal or parietal lobes, traveling between the superior sagittal sinus (SSS) and the sylvian fissure. The vein of Labbe (VL), also known as the inferior anastomotic vein, is the largest vein crossing the temporal lobe between the transverse sinus (TS) and sylvian fissure. The superficial Sylvian vein (SSV) or superficial middle cerebral vein, arises at the posterior end of the sylvian fissure and courses anteroinferiorly along the fissure. It receives venous blood from the fronto-sylvian, parieto-sylvian, and temporo-sylvian veins, and generally anastomoses with the VT and VL. It generally empties into the venous sinuses along the sphenoid ridge. These three largest veins are generally

From the Department of Neurosurgery, Montreal Neurological Institute and Hospital, McGill University, Montreal, Quebec, Canada.

Received June 30, 2008. Final Revisions Submitted September 15, 2008. Correspondence to: Taner Tanriverdi, Department of Neurosurgery, Montreal, Neurological Institute and Hospital, 3801 rue University, suite 109, Montreal, Quebec H3A 2B4, Canada. 
encountered during fronto-parieto-temporal craniotomies, which are the commonest surgical approaches in neurosurgery. Thus, practical knowledge about the course of these veins is utmost importance for neurosurgeons during surgical procedures in order to avoid serious complications. The aim of this study is to document the course of SAVs that would be of practical use in the operating room. Furthermore, the relation of VT and VL to the central area and the differences regarding the dominance of these veins according to the sex and dominant hemispheres are also provided.

\section{Materials AND Methods}

The present clinical study included 251 patients who underwent fronto-parieto-temporal craniotomy due to intractable temporal lobe epilepsy at the Montreal Neurological Institute (MNI) between 1972 and 1987. The patients presented here had fronto-parieto-temporal craniotomies which were large enough to show the courses of the VT, VL, and SSV. The cortex was electrically stimulated to provide anatomic location of the sensorimotor strip, thus electrical cortical stimulation permitted us to study the relation between the courses of the VT/VL and the central area more accurately. The courses and relationship between SAVs and the central area were based on intraoperative photograph findings. All patients had cerebral angiography before surgery in order to visualize the vessels and the dominant hemispheres were determined by sodium amytal test (Wada test) ${ }^{17}$.

\section{Statistical Analysis}

All data collected from each patient were organized in a database (Excel, Microsoft Corp.) Numeric variables were provided as the mean \pm SD. For statistical analysis, we used chisquare $\left(\chi^{2}\right)$ analyses to compare the relevant groups. Correlations were made using Pearson's correlation tests. A probability value $<0.05$ was considered statistically significant. All statistical calculations were performed using commercially available software. (SPSS version 14.0 SPSS Inc.)

\section{RESULTS}

\section{General characteristics of patients}

This study included 118 males and 133 females with a mean age of $25.04 \pm 8.6$ years. All underwent corticoamygdalohippocampectomy due to medically-refractory temporal lobe epilepsy. The surgical technique was reported elsewhere in the literature $^{18}$. Table 1 provides a summary of clinical characteristics of the patients. The right-sided and left-sided craniotomies were performed in 125 and 126 patients, respectively. In 209 patients $(83.2 \%)$, sodium amytal test showed left hemispheric dominance in 37 patients $(14.7 \%)$. Bilateral dominance was seen in only 5 patients $(2 \%)$.

\section{General characteristics of superficial anastomotic veins}

The most common predominant veins seen over the lateral hemisphere was the combination of the VL and SSV (19.5\%), followed by the combination of the VL and VT (15.5\%), and VT and SSV (11.5\%) (Figure 1 through 3). In 28 patients (11.2\%), all three SAVs were predominant (Figure 4) and in $15(6 \%)$ cases no predominant vein was detected. Individually, the VT $(13.1 \%)$
Table 1: Patients' characteristics included in this study

\begin{tabular}{ll|ll}
\hline Factors & Value & Male & Female \\
\hline Mean age (years) & $25.04 \pm 8.6$ & $25.4 \pm 9.4$ & $24.7 \pm 7.9$ \\
Male/Female & - & 118 & 133 \\
Handedness (Right/Left/Both) & $193 / 48 / 10$ & $85 / 26 / 7$ & $108 / 22 / 3$ \\
Seizure onset (years) & $9.74 \pm 8.4$ & $10.0 \pm 8.4$ & $9.4 \pm 8.4$ \\
Mean duration of seizure (years) & $15.4 \pm 8.4$ & $15.6 \pm 8.6$ & $15.2 \pm 8.2$ \\
Dominant hemisphere (Right/Left/Bilateral) & $37 / 209 / 5$ & $20 / 96 / 2$ & $17 / 113 / 3$ \\
Hemisphere operated (Right/Left) & $125 / 126$ & $56 / 62$ & $69 / 64$ \\
Surgery type (CorAH) & 251 & - & - \\
\hline
\end{tabular}

CorAH: Cortico amygdalohippocampectomy

and SSV $(13.1 \%)$ were predominated in equal number of patients and in 25 patients $(10 \%)$, VL was the predominant vein. Statistical analysis showed that there were no differences among the vein groups regarding dominance $\left(\chi^{2} ; \mathrm{p}=0.16\right)$. A total of 25 patients $(9.9 \%)$ showed duplication of any of the three veins.

Although the VT showed variations in its course, generally it directed posteriorly and superiorly to join the SSS. Its lower end usually was a single channel which anastomosed with the veins

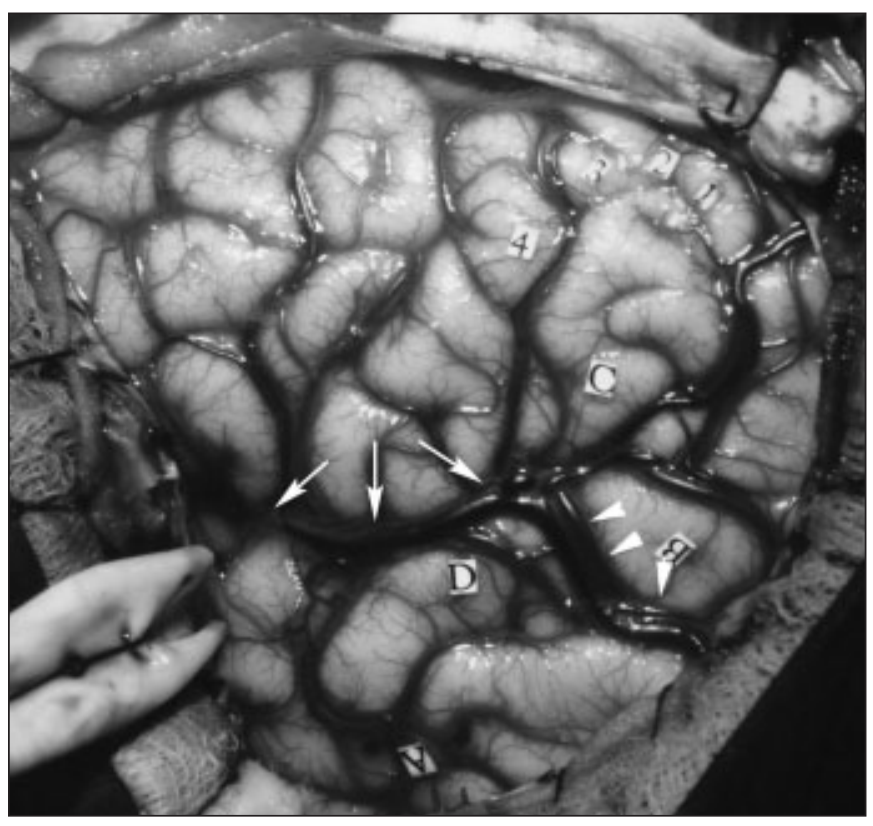

Figure 1: Codominance of the vein of Labbe (white arrow heads) and the superficial sylvian vein (white arrows). This drainage pattern was the most common and seen in $19.5 \%$ of the cases. 


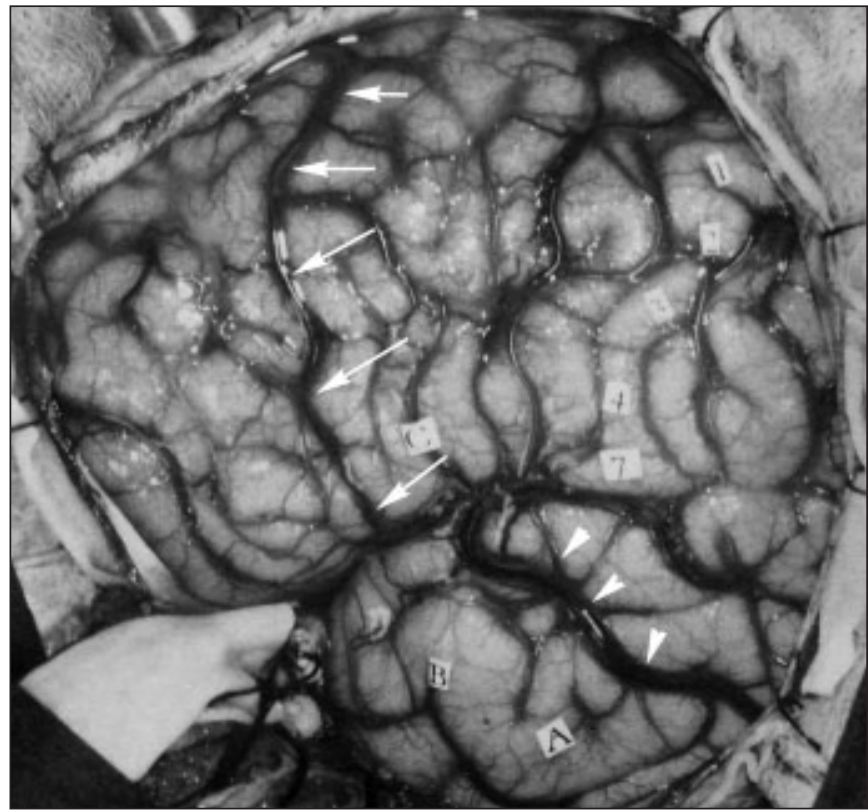

Figure 2: The veins of Trolard (white arrows) and Labbe (white arrow heads) are dominant. This pattern drainage was the second most common and seen in $15.5 \%$ of the cases.

along the sylvian fissure but in some cases it divided into multiple channels that joined the SSV. In this study the VT was most commonly located at the level of the central vein $(\mathrm{CV})$ $(14.3 \%)$. In 14 cases $(5.5 \%)$, the VT showed duplication in which two dominant veins of almost similar size crossed the interval between the SSV and SSS.

In almost all cases, if present, the VL originated from the SSV and was directed posteriorly and inferiorly to join the transverse sinus. The VL was most commonly located at the level of the middle temporal vein (MTV), which was seen in 94 cases $(37.5 \%)$. In some cases we found a double VL, in which the anterior one was almost always larger than the posterior.

In the 251 craniotomies examined in this study, the SSV was seen in 178 cases $(70.9 \%)$ and usually showed constant course. It originated at the posterior end of the sylvian fissure and coursed anteriorly and inferiorly between the lips of the fissure. In this study SSV was dominant in $33(13.1 \%)$ cases and showed duplication in six cases in which the superior one (frontal site) is larger than the inferior one (temporal site).

\section{Superficial anastomotic veins according to sex and side of hemisphere}

In 118 male patients, $56(47.4 \%)$ and $62(52.5 \%)$ right and left hemispheres were operated, respectively. The VT predominated on the right side and the VL predominated on the left side. Interestingly, in female patients the VT predominated on the left hemisphere. As opposed to males, the VL and SSV were dominant in the right hemispheres. Comparing the total number of the predominant veins regarding the $\operatorname{sex}\left(\chi^{2} ; p>0.05\right)$ or side of the hemisphere in male and female patients $\left(\chi^{2} ; p>0.05\right)$ did not reveal statistically significant differences.

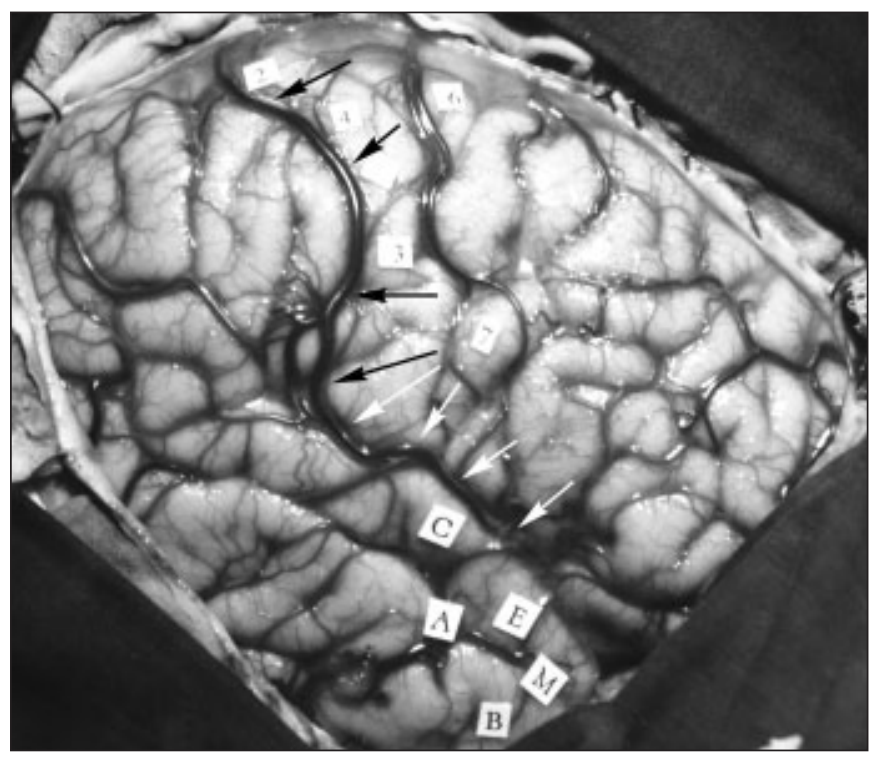

Figure 3: The veins of Trolard (black arrows) and superficial sylvian vein (white arrows) are dominant. This pattern of drainage was the third most common and seen in $11.5 \%$ of the cases.

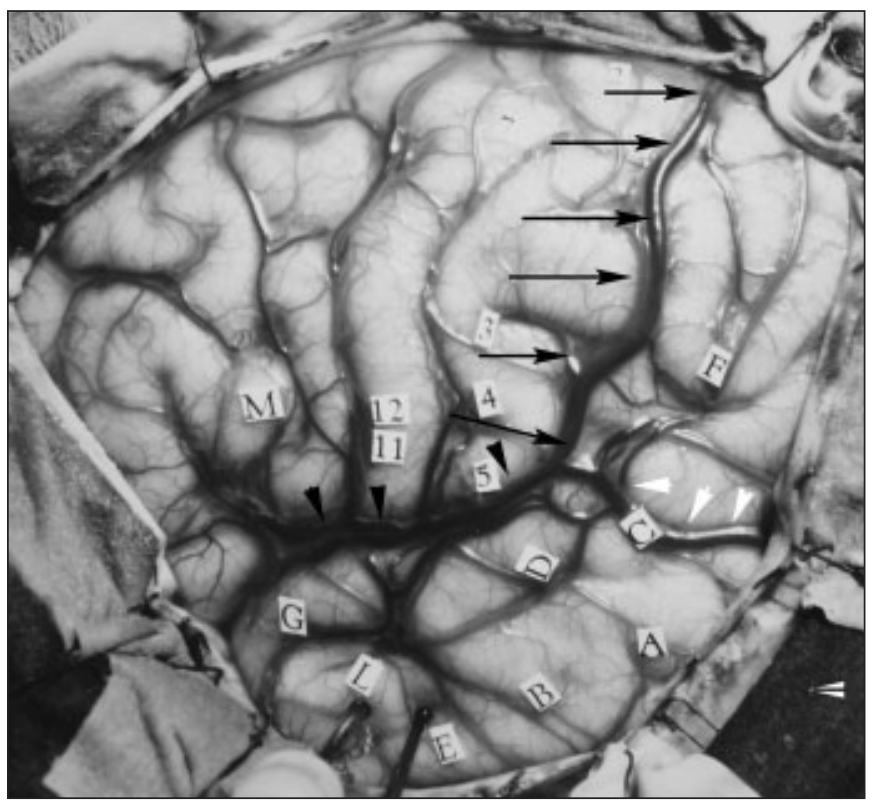

Figure 4: All three anastomotic veins are present. This type was seen in $11.2 \%$ of the cases. Black arrows: vein of Trolard; white arrow heads: vein of Labbe and white arrow heads: superficial sylvian vein. 


\section{Superficial anastomotic veins according to the sex and dominant hemisphere}

In 118 males, sodium amytal test showed that the right hemisphere was dominant in $20(16.9 \%)$, while the left hemisphere was dominant in $96(81.3 \%)$ patients. Two cases (1.6\%) had bilateral hemispheric dominance. In 133 female patients, the right and left hemispheres were dominant in 17 $(12.7 \%)$ and $113(84.9 \%)$ patients, respectively and three $(2.2 \%)$ had bilateral dominance.

In the group of 20 male patients with right hemispheric dominance, the most common predominant veins were found to be the codominance of the VL + VT and VL + SSV. Statistical analysis showed no significant difference regarding the dominance of the veins in male patients with right hemispheric dominance $\left(\chi^{2} ; p>0.05\right)$. However; in the group of 96 male patients with left hemispheric dominance, the codominance of the VL and SSV was the most common. Again, no significant differences among the veins regarding predominance in male patients who had left hemispheric dominance was found $\left(\chi^{2} ; \mathrm{p}>\right.$ $0.05)$.

In the group of 17 female patients with right hemispheric dominance, the VT was predominant in 3 cases $(17.6 \%)$, followed by SSV (11.7\%) and VL (5.8\%). There were no significant differences among the veins regarding predominance in female patients with right hemispheric dominance $\left(\chi^{2} ; \mathrm{p}>\right.$ $0.05)$. In 113 female patients with left hemispheric dominance, the vein of Labbe and SSV combination was the most predominant type. Similar to patients with right hemispheric dominance, no statistically significant differences were found regarding predominant vein groups in females with left hemispheric dominance $\left(\chi^{2} ; \mathrm{p}>0.05\right)$.

\section{Relation of the Veins of Trolard and Labbe to the central area}

In this study the relationship between the VT and central area was determined by its course at a site that would correspond to the PreCV, CV, or PostCV. And the relationship between the VL and the lower central area was determined by closeness of its origin to the lower central area such as precentral gyrus (PreCG), pli de passage and postcentral gyrus (PostCG) (Figure 5). In 226 patients the VT and/or VL showed relationships to the central area (Table 2). As can be seen in Table 2, the VT was associated with the central area in $39.8 \%$ of the cases and the VL was associated with the lower central area in almost $50.1 \%$ of the cases. Although speculative, these results might show that in $50 \%$ of the cases, tracking the VL may guide us to the lower central area, or with the same context in almost $40 \%$ of cases, the VT may help us to identify the central area.

\section{DisCuSSION}

The main findings which emerged from this study are that: 1) regardless of sex and side of hemispheric dominance, the most common predominant SAV was the combination of the VL and SSV; 2) the VT showed variable courses relative to the VL and SSV; 3) VT was most frequently found at the level of the central vein and the VL was located frequently at the level of the MTV; 4) regardless of sex, the predominant superficial venous channel in the right hemisphere was the SSV, while the VL predominated in the left.

The intracranial venous system has been extensively studied and its variations in course, size, and collaterals have been documented in cadaveric ${ }^{1-8}$ and angiographic studies ${ }^{9-15}$. However; these studies have provided different findings with respect to the anatomy of the intracranial veins, especially cortical veins, regarding drainage patterns, size, or collaterals. Thus, there are considerable variations in the individual cortical veins, not only in different brains but also from either side in the same brain.

Little attention has been paid to the SAVs from a neurosurgeon's perspective. There is no doubt that several catastrophic complications after a neurosurgical procedure are related to iatrogenic venous damage. Since the fronto-temporoparietal approach is one of the most common surgical openings in neurosurgery, a good knowledge of the superficial anastomotic venous anatomy is of prime importance for neurosurgeons, especially in epilepsy surgery.

Reviewing angiographic and cadaveric studies showed variations in size and predominant pattern of drainage of the three SAVs, especially in the drainage pattern of the VT and VL. Previous angiographic studies claimed that the distribution of superficial cortical veins is not as irregular as supposed and examination of the venous phase of cerebral angiography might

Table 2: Relations of the veins of Trolard and Labbe to the central area

\begin{tabular}{lccccccc}
\hline Factors & PreCV & $\begin{array}{l}\text { Central } \\
\text { vein }\end{array}$ & PostCV & PreCG & $\begin{array}{l}\text { Pli de } \\
\text { passage }\end{array}$ & PostCG & Total (\%) \\
\hline Trolard & 31 & 39 & 30 & & & & $100(39.8)$ \\
Labbe & & & & 38 & 20 & 68 & $126(50.1)$ \\
Valid \% & 10.8 & 14.3 & 10.4 & 15.1 & 8.0 & 26.7 & $226(90.0)$ \\
\hline
\end{tabular}

PostCG: Postcentral Gyrus; PostCV: Postcentral vein; PreCG: Precentral Gyrus; PreCV: Precentral vein 


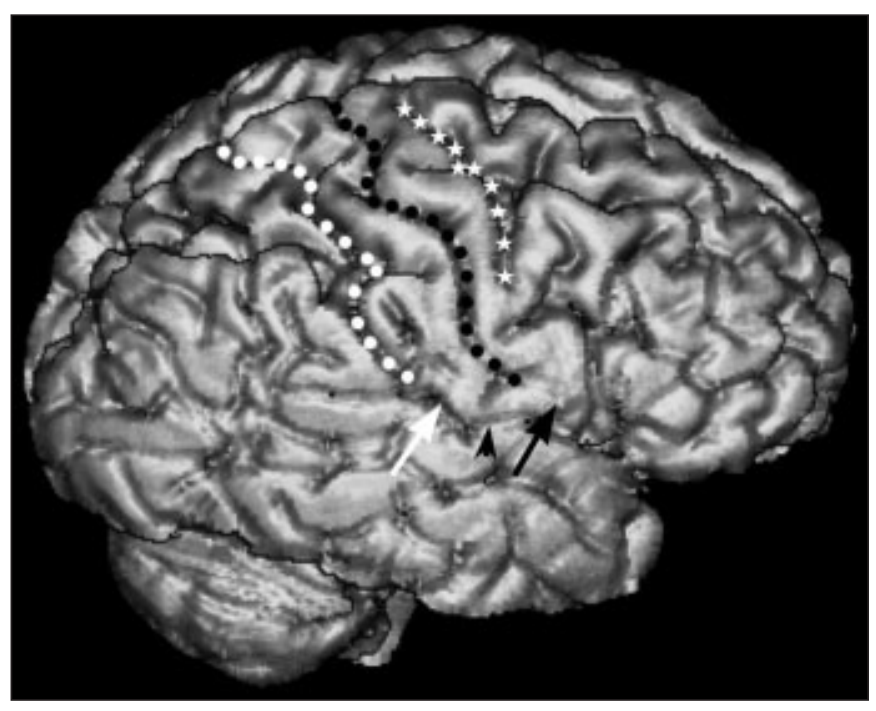

Figure 5: Illustrative 3D image. The relationship between the vein of Trolard and the central area is defined depending on the course(s) of the vein of Trolard at the level of the precentral sulcus (white stars), central sulcus (black dots), and postcentral sulcus (white dots). The relationship between the vein of Labbe and the lower central area is defined depending on the origin from the superficial sylvian vein close to precentral gyrus (black arrow), pli de passage (black arrow head), and postcentral gyrus (white arrow).

provide information in localizing expanding lesions by revealing poor filling and displacement of direction. In Gvozdanovic's ${ }^{11}$ series of 500 phlebograms, it has been shown that the drainage of the convexity in the direction of the superior sagittal, transverse, and cavernous sinuses was fairly regular and that the ascending veins were fairly equally distributed ${ }^{11}$. According to Gvozdanovic $^{11}$, cases of domination of the drainage in one direction through one of the three largest SAVs represented only extreme forms of one of the possible variations. However; Matsubara $^{12}$, depending on 117 normal and 117 pathologic phlebograms stated that, in most cases, it was easy to find out one direction of domination of drainage from the one of the three SAVs. In this study, variations in SAVs were seen in $30 \%$ of cases and the majority had domination drainage through the VT. In contrast, variations in pathologic phlebograms were seen in $54 \%$ and the marked increases in SSV and VL were noticed compared with normal cases and, following the removal of the expanding lesion, abnormal drainage patterns became normal in some cases. Thus, this study concluded that drainage through the SSV and VL was really pathologic. Furthermore, Matsubara ${ }^{12}$ found that domination of drainage through the VT was in most of the normal cases, thought it was found mostly in the right hemisphere of females between 10 to 19 years-of-age and also in the right hemisphere of males between 20 to 29 years-of-age, suggesting that the differences in cortical venous return in both hemispheres have some relation with the differences in functions of the two hemispheres in these age groups. In their phlebograms of 175 brains, Delmas, et $\mathrm{al}^{19}$ observed that the VL was predominant on the left side in $42 \%$ of cases and on the right side in $21 \%$, whereas the VT was predominant on the right side in $52 \%$ and on the left side in $24 \%$. In the study of 180 normal carotid angiograms, Di Chiro ${ }^{13}$ has noted that, in the majority of cases, the VL was the most common predominant vein (40\%), followed by the VT (32\%). Codominance of both the VT and VL was seen in $11 \%$. Di Chiro ${ }^{13}$ also noted that most frequently, the VT was found at the level of the CV and sometimes the double VT drained towards the SSS with approximately the same caliber. Furthermore, the VT predominated in the nondominant hemisphere and the VL predominated in the dominant hemisphere. Moreover, there was an inverse relationship between the veins of Trolard and Labbe. When one predominated, the other on that side was generally small or absent.

Cadaveric studies also demonstrated different findings regarding the SAVs. Wolf et $\mathrm{al}^{20}$ noted that the superficial cortical veins have a relatively constant appearance, location, and number and the most variable vessel was the SSV. In 1958, Perese $^{6}$, depending on 100 autopsy findings, reported that the temporal and occipital veins are made up of the so called vein of Labbe which consisted of two or more branches originating in or below the sylvian fissure. These vessels joined the lateral occipital veins at the preoccipital notch and entered the tentorium to drain into the transverse sinus. Furthermore, the SSV was composed of two or three branches. However; in ten cadaveric studies, Oka et $\mathrm{al}^{7}$ showed that the SSV usually arises as a single channel and it receives fronto-sylvian, parietosylvian, and temporo-sylvian veins. When the central segment of the vein is absent the anterior segment joins the sphenoparietal sinus and the posterior segment joins the VT and VL. According to these authors ${ }^{7}$, the VT is mostly found at the level of the PostCV and its lower end is usually a single channel that anastomoses with the veins along the sylvian fissure. It sometimes splits on the lower part of the frontal and parietal convexity into multiple channels that join the SSV. In some specimens, the VT may duplicate into two large veins of similar size. In 12 of 20 hemispheres, they found that the VL was found at the level of the MTV and in some cases they found double VL in which the posterior one was usually larger. In agreement with Oka et $\mathrm{al}^{7}$, Ciszek et $\mathrm{al}^{8}$ in 56 cadaveric hemispheres showed that the SSV was present as a single trunk in $85 \%$ and during its course several tributaries run from the temporal and frontal lobes and joined in one trunk just before its opening into the sphenoparietal sinus. In 82 and $58 \%$ of the cases the SSV anastomosed with the VL and VT, respectively. In $40 \%$ SSV anastomosed with both veins.

In recent years studies using magnetic resonance (MR) venograms showed somewhat different results compared to both cadaveric and cerebral venographic studies mentioned above. In 100 MR venograms Ayanzen et al ${ }^{10}$ showed that there are significant differences between the right and left hemispheres regarding the predominant SAVs. In this study the VT predominated in 37 and $34 \%$ of the cases in the right and left hemispheres, respectively. On the other hand, the VL predominated in $91 \%$ of the cases in the right and $96 \%$ of the cases in the left hemisphere. Similar results have been demonstrated in a MR venogram study of 100 patients by Surendrababu et $\mathrm{al}^{14}$. They showed that the relatively constant 
superficial cerebral vein identified was the VT, which was observed in 79 patients in the right and 75 patients in the left hemisphere. Similarly the VL was found in 70 patients in the right and 68 patients in the left hemisphere. In a recent study using three-dimensional contrast-enhanced magnetizationprepared rapid gradient-echo in 400 hemipsheres (200 patients), Ikushima et $\mathrm{al}^{15}$ showed that the most common dominant vein was the VL. Undeveloped VL was seen in $16 \%$ and in the cases with the dominant VT, it commonly located at the level of the PostCV. They also noted that the VT and SSV predominated on the right side whereas the VL predominated on the left side. The VL showed a few variations relative to the VT and SSV.

\section{Present study}

The authors in this study underline that the aim here is not to provide detailed anatomy of the cortical cerebral veins. We simply wanted to to report courses of SAVs seen during frontotemporo-parietal craniotomies. Furthermore, we did not have a chance to compare the right and left hemispheres regarding the three largest SAVs in the same individual. However; we think that our intraoperative findings would be helpful for neurosurgeons while performing surgery to recognize the SAVs and the relationship with the central area.

Our results in this study are in line with the majority of previous studies; however, some differences were found. In contrast to Gvozdanovic ${ }^{11}$ and Wolf et $\mathrm{al}^{20}$, but similar to Di Chiro $^{13}$, drainage patterns of SAVs in our surgical series showed variations and the VT showed different courses relative to the VL and SSV. Regardless of the sex and the side of the cerebral hemisphere, the codominance of the VL and SSV was the most common predomninant vein, as has been substantiated by Ciszek et $\mathrm{al}^{8}$. We do not agree with Matsubara ${ }^{12}$ that direction through the VL and/or SSV is pathologic. In line with Di Chiro ${ }^{13}$ but different from Oka et $\mathrm{al}^{7}$, and Ikushima et $\mathrm{al}^{15}$ we found that the VT were frequently located at the level of the CV. The VL coursed in the majority of the cases at the level of the MTV as has been found by Oka et $\mathrm{al}^{7}$. Different from Di Chiro ${ }^{13}$ and Peresa $^{6}$, the VL and SSV did not show frequent duplications and they did not have two or more branches in our study and the SSV coursed as a single vein in the majority of the cases as shown by Oka et $\mathrm{al}^{7}$. Regradless of the sex and the hemispheric dominance, the combination of the VL and SSV was the most common predominant vein in either the right or the left hemisphere, a finding different from previous studies. Similarly, regardless of the sex, the combination of the VL and SSV was the most common predominant vein in the dominant hemisphere (right or left), also a finding different from the previous studies. Depending on our own results and others, we support the common notion that there are considerable variations in the courses of SAVs and these variations make it difficult to define a normal venous pattern on the lateral surface of the brain.

Our study showed that in $36.2 \%$ of the cases, a single vein predominates and drains most of the lateral surface of the cerebral hemisphere. Thus, sacrificing or damage to one of these predominant veins may cause significant postoperative morbidity and even mortality in at least $36 \%$ of the cases. Superficial cortical veins should not be sacrificed if the vein seems to be larger than normal because increased size may indicate that the vein drains a larger area than normal and increases the likelihood of complication if it is sacrified.

The superficial anastomotic veins showed considerable variations in their courses and it is difficult to define an exact pattern although some courses showed constant direction. Attention should be paid not to damage these veins since in a considerable number of cases a single dominant vein may be responsible for the draining of the majority of the surface of cerebral hemisphere. Familiarity with the surface anatomy of the SAVs is of utmost importance for all neurosurgeons because awareness and identification of the predominant venous complexes during surgery is the first step in avoiding devastating complications.

\section{ACKNOWLEDGMENTS}

We greatly appreciate the valuable help of Monika Malecka and Luisa Birri in collecting the data from the archive and Fusun Kobas Tanriverdi for her technical help. T.T. is a fellow at the Department of Neurosurgery (section of Epilepsy Surgery), Montreal Neurological Institute, Montreal, Quebec, Canada and supported by a scholarship provided by the Mark Rayport \& Shirley Ferguson Fund.

This study was supported partly by the grants from the Savoy Foundation.

\section{REFERENCES}

1. Koperna T, Tschabitscher M, Knosp E. The termination of the vein of Labbe and its microsurgical significance. Acta Neurochir (Wien). 1992;118:172-5.

2. Meder J-F, Chiras J, Roland J, Guinet P, Bracard S, Bargy F. Venous territories of the brain. J Neuroradiol. 1994;21:118-33.

3. Guppy KH, Origitano TC, Reichman OH, Segal S. Venous drainage of the infralateral temporal lobe in relationship to transtemporal/ transtentorial approaches to the cranial base. Neurosurgery. 1997;41:615-20.

4. Han H, Tao W, Zhang M. The dural entrance of cerebral bridging veins into the superior sagittal sinus: an anatomical comparison between cadavers and digital substraction angiography. Neuroradiology. 2007;49:169-75.

5. Han H, Yao Z, Wang H, Deng X, Fong AHY, Zhang M. Dural entrance of the bridging vein into the transverse sinus provides a reliable measure for preoperative planning: an anatomic comparison between cadavers and neuroimages. Neurosurgery. 2008;62[ONS Suppl 2]:)ONS289-ONS296.

6. Perese DM. Superficial veins of the brain from a surgical point of view. J Neurosurg. 1960; 17:402-12.

7. Oka K, Rhoton AL, Barry M, Rodriguez R. Microsurgical anatomy of the superficial veins of the cerebrum. Neurosurgery. 1985; 17:711-48.

8. Ciszek B, Dabrowska M, Andrzejczak A, Aleksandrowicz R, Mazurowski W, Zabek M. Middle superficial cerebral vein. Folia Morphol (Warsz). 1998;57:149-55.

9. Hochberg FH, May ML. Arteriographic correlates of handedness. Neurology. 1975;25:218-22.

10. Ayanzen RH, Bird CR, Keller PJ, McCully FJ, Theobald MR, Heiserman JE. Cerebral MR venography: normal anatomy and potential diagnostic pitfalls. AJNR Am J Neuroradiol. 2000;21: 74-8.

11. Gvozdanovic V. Changes in the superficial veins in cases of intracranial expanding processes. Acta Radiologica. 1956;46: 195-202.

12. Matsubara T. An observation on cerebral phlebograms with special reference to the changes in the superficial veins. Nagoya J Med Sci. 1960;23:86-100.

13. Di Chiro G. Angiographic patterns of cerebral convexity veins and superficial dural sinuses. Am J Roentgenol. 1962;87:308-21. 
14. Surendrababu NRS, Livingstone SRS. Variations in the cerebral venous anatomy and pitfalls in the diagnosis of cerebral venous sinus thrombosis: low field MR experience. Indian J Med Sci. 2006;60:135-42.

15. Ikushima I, Korogi Y, Kitajima M, Yamura M, Yamashita Y. Evaluation of drainage patterns of the major anastomotic veins on the lateral surface of the cerebrum using three-dimensional contrast-enhanced MP-RAGE sequence. Eur J Radiol. 2006;58: 96-101.

16. Aydin IH, Tuzun Y, Takci E, Kadioglu HH, Kayaoglu CR, Barlas E. The anatomical variations of sylvian veins and cisterns. Minim Invas Neurosurg. 1997;40:68-73.
17. Wada J, Rasmussen T. Intracarotid injection of sodium amytal for lateralization of cerebral speech dominance. J Neurosurg. 1960; 17:266-282.

18. Olivier A. Surgery of epilepsy: methods. Acta Neurol Scand Suppl. 1988;117:103-13.

19. Delmas A, Pertuiset B, Bertrand G. Les veines du lobe temporal. Rev Otoneuroophtalmol. 1951;23:224-30.

20. Wolf BS, Huang YP, Newman CM. The superficial sylvian venous drainage system. Am J Roentgenol Radium Ther Nucl Med. 1963;89:398-410. 